\title{
Questes
}

\section{Le temps de la fête}

\section{La fête de la Croix : unité du culte, pluralité des fêtes}

\section{Céline Ménager}

\section{(2) OpenEdition}

1 Journals

\section{Édition électronique}

URL : http://journals.openedition.org/questes/4276

DOI : 10.4000/questes.4276

ISSN : 2109-9472

\section{Éditeur}

Les Amis de Questes

\section{Édition imprimée}

Date de publication : 31 octobre 2015

Pagination : 63-80

ISSN : 2102-7188

\section{Référence électronique}

Céline Ménager, «La fête de la Croix : unité du culte,

pluralité des fêtes », Questes [En ligne], 31 | 2015, mis en ligne le 24 janvier 2016, consulté le 20 avril 2019. URL : http://journals.openedition.org/questes/4276 ; DOI : 10.4000/questes.4276 


\section{La fête de la Croix : unité du culte, pluralité des fêtes}

\section{Céline Menager}

Université Paris-Sorbonne

Dans les premiers siècles de notre ère, l'association entre le christianisme et la croix ne va pas de soi. Il fallut en effet plusieurs siècles et un véritable retournement des mentalités pour faire d'un symbole d'infamie la marque de la religion triomphante ${ }^{1}$. Ce processus est marqué par plusieurs événements survenus au début $\mathrm{du}_{\mathrm{IV}}^{\mathrm{e}}$ siècle, principalement sous le règne de Constantin. Citons tout d'abord la bataille du Pont Milvius qui consacre la domination du nouvel empereur Constantin sur l'Ouest de l'empire. Les récits de cet épisode sont nombreux et les variantes multiples. L'essentiel pourrait être résumé ainsi: au cours d'un songe, Constantin voit apparaître une croix accompagnée de la phrase «hoc signum vinces », symbole que le Christ lui ordonne de faire peindre sur les boucliers de ses soldats pour obtenir la victoire ${ }^{2}$. Sous le même Constantin, la crucifixion cesse d'être utilisée

\footnotetext{
${ }^{1}$ Henri Leclercq, «Croix et crucifix », dans Dictionnaire d'archéologie chrétienne et de liturgie, éd. Henri Leclercq et Fernand Cabrol, Paris, Letouzey et Ané, 1914, vol. III, part. 2, col.3045-3131. Louis van Tongeren, Exaltation of the Cross : toward the origins of the feast of the Cross and the meaning of the Cross in early Medieval liturgy, London, Peeters, coll. «Liturgia condenda », $\mathrm{n}^{0} 11,2000$, p. 2-4. Paul Veyne, Quand notre monde est devenu chrétien (312-394), Paris, Albin Michel, coll. «Bibliothèque Albin Michel, Idées », 2007, p. 50-52.

${ }^{2}$ La bibliographie sur l'empereur Constantin est pléthorique. Pour une approche synthétique, on pourra consulter deux biographies récentes: Bertrand Lançon et Tiphaine Moreau, Constantin: Un Auguste chrétien, Paris, Armand Colin, coll. «Nouvelles biographies historiques », 2012; Pierre Maraval, Constantin le Grand : empereur romain, empereur chrétien (306-337), Paris, Tallandier, 2011.
} 
par la justice comme châtiment ${ }^{3}$. À la génération suivante, sous l'empereur Constance, une croix lumineuse apparaît dans le ciel de Jérusalem ; l'événement est rapporté à l'empereur par l'évêque Cyrille qui y voit une façon pour Dieu de favoriser Constance comme il avait déjà favorisé Constantin ${ }^{4}$. L'association entre Constantin et le symbole de la Croix devient rapidement si étroite que c'est à sa mère que l'on attribua tout naturellement dès la fin $\mathrm{du} \mathrm{IV}^{\mathrm{e}}$ siècle, la redécouverte de la précieuse relique du bois de la Croix ${ }^{5}$.

$\mathrm{Au}$ cours du haut Moyen Âge, nous voyons émerger plusieurs fêtes célébrant la Croix. Les principales, sur lesquelles portera notre étude, sont l'adoration du Vendredi saint et les fêtes de l'exaltation et de l'invention de la Croix, respectivement les 14 septembre et 3 mai. Les deux premières célébrations apparaissent à Jérusalem avant de passer en Occident, tandis que la troisième est une création entièrement occidentale, voire romaine.

Depuis le début $\mathrm{du} \mathrm{XX}^{\mathrm{e}}$ siècle, ethnologues et folkloristes ont cherché à établir un lien entre les cultes chrétiens et les mythes antiques. Ainsi Pierre Saintyves ${ }^{6}$ a pu voir dans les saints les successeurs des

\footnotetext{
${ }^{3}$ Henri Leclercq, «Croix et crucifix », art. cit. ; Paul Veyne, Quand notre monde est devenu chrétien, op. cit.

${ }^{4}$ Henri Leclercq, «Croix (invention et exaltation de la vraie) », dans Dictionnaire d'archéologie chrétienne et de liturgie, éd. Henri Leclercq et Fernand Cabrol, Paris, Letouzey et Ané, 1914, vol. III, part. 2, col.3131-3139, cit. col. 3136. Lettre de Cyrille à Constance II en 351, PG XXXIII, 1167.

5 Sur la vie de l'impératrice Hélène (vers 250-327/8) et sur l'émergence de sa légende, on consultera: Jan Willem Drijvers, Helena Augusta: the mother of Constantine the Great and the legend of her finding of the true cross, Leiden, E. J. Brill, coll. "Brill's studies in intellectual history», $\mathrm{n}^{\circ}$ 27, 1992. Sur le second point on se référera également à Stephan Borgehammar, How the Holy Cross was found: from event to medieval legend, Stockholm, Almqvist and Wiksell, coll. «Bibliotheca theologiae practicae », $\mathrm{n}^{\circ}$ 47, 1991. Hans A. Pohlsander, Helena: Empress and Saint, Chicago, Ares Publishers Inc., 1995

${ }^{6}$ Pierre Saintyves, «De la méthode dans l'étude du folklore chrétien », Revue de l'histoire des religions, $\mathrm{n}^{\mathrm{o}} 88,1923, \mathrm{p} .151-163$.
} 
dieux gréco-romains tandis que Henri Dontenville ${ }^{7}$ puisait dans le fond celtique. Pas plus tard qu'en 2003, Philippe Walter proposait de relire le calendrier des saints à la lumière d'un cycle carnavalesque d'origine indo-européenne ${ }^{8}$.

Si la démarche de recherche des origines n'est pas sans intérêt pour comprendre les logiques de la christianisation du calendrier occidental, elle nous fait courir le risque d'oublier les besoins des hommes du Moyen Âge au profit d'un Homme immuable. C'est aussi méconnaître le caractère profondément subversif du christianisme, capable de nombreux retournements. Nous évoquions le changement autour du symbole de la Croix. Un autre renversement, plus fondamental, est le statut des morts : relégués à l'extérieur de la cité chez tous les peuples préhistoriques puis antiques, les morts trouvèrent droit de cité au cœur de la ville et jusque dans les églises grâce au culte des saints. Le christianisme se joue ainsi de la frontière la plus hermétique posée par l'Antiquité' .

Claude Lévi-Strauss disait, évoquant la figure du père Noël, que nulle célébration ne saurait survivre si elle ne remplissait pas une fonction fondamentale ${ }^{10}$. C'est à la lumière de cette tension entre origine

7 Henri Dontenville, Mythologie française, Paris, Payot, coll. «Le regard de l'histoire », $1973^{2}$ (1948).

${ }^{8}$ Philippe Walter, Mythologie chrétienne: Fêtes, rites et mythes du Moyen Âge, Paris, Imago, coll. «Le grand livre du mois », 2003, p. 27-42.

${ }^{9}$ Peter Brown, Le Culte des saints : son essor et sa fonction dans la chrétienté latine, trad. Aline Rousselle, Paris, Éditions du Cerf, coll. «Histoire», $\mathrm{n}^{\circ}$ 2, 1996. Michel Lauwers, Naissance du cimetière. Lieux sacrés et terre des morts dans l'Occident médiéval, Paris, Aubier, coll. «Collection historique », 2005.

${ }^{10}$ Les explications par survivances sont toujours incomplètes, car les coutumes ne disparaissent ni ne survivent sans raison. Quand elles subsistent, la cause se trouve moins dans la viscosité historique que dans la permanence d'une fonction que l'analyse du présent doit déceler »: Claude Lévi-Strauss, «Le Père noël supplicié », Temps modernes, 77, 1952, p. 1572-1590, cit p. 1584, cité par Antoinette GlauserMatecki, Un Aspect du cycle de mai en Europe occidentale : rites et coutumes des calendes de mai, thèse de l'EHESS, 1996, cit. p. 1. 
et sens médiéval que nous tenterons de comprendre les fêtes de la Croix. Ces différentes fêtes nous invitent à observer les phénomènes de transfert qui existent entre l'Orient et l'Occident et à nous interroger sur les rôles de Jérusalem et de Rome dans l'élaboration du calendrier festif entre la fin de l'Antiquité et l'époque carolingienne, où les dates de la plupart des fêtes actuelles sont fixées. L'importance de la Croix dans la conversion de l'empire questionne également le rôle de l'empereur dans ces célébrations. On pourrait ainsi envisager que la multiplication des fêtes repose sur un partage du sacré entre la hiérarchie ecclésiastique et l'empereur. Mais la chute de l'Empire en Occident rend vite l'hypothèse obsolète. Nous nous concentrerons donc sur le rôle que jouent ces fêtes dans la christianisation des pratiques occidentales. Nous aborderons tour à tour trois fêtes du calendrier romain : le Vendredi saint, l'Exaltation de la Croix et l'Invention de la Croix.

\section{Le Vendredi saint. Dynamique d'une fête chrétienne}

La première et la plus connue de ces célébrations est l'adoration de la Croix qui avait lieu à Jérusalem, au cours de la semaine sainte, le vendredi, et qui consistait surtout dans l'exposition des reliques de la Croix aux yeux des fidèles. La fête apparaît à Jérusalem. Elle est décrite pour la première fois, dans les années 380, dans le Journal d'Égérie ${ }^{11}$. Il s'agit d'une lettre dans laquelle une abbesse originaire de Gaule raconte à ses sœurs restées au pays son voyage en Terre sainte et en Égypte. Elle y détaille tous les monuments qu'elle a visités et, bien que le texte soit lacunaire, elle nous donne de précieuses indications sur les célébrations qui avaient lieu à cette époque à Jérusalem. Elle décrit en particulier le défilé des pèlerins devant un morceau des reliques de la vraie Croix lors

${ }^{11}$ Égérie, Journal de voyage : itinéraire, éd. Pierre Maraval, Paris, Éditions du Cerf, coll. «Sources chrétiennes », nº 296, 1982. 
du Vendredi saint ${ }^{12}$. La cérémonie a dû se mettre en place entre les années 330, date supposée de la redécouverte de la vraie Croix, et l'époque d'Égérie où la pratique semble bien ancrée ${ }^{13}$. Cette fête est rendue possible par la - supposée - redécouverte de la Croix, mais aussi par le retournement de la valeur de ce symbole que nous évoquions plus haut.

Peu après Égérie, on trouve un autre témoignage sur cette cérémonie sous la plume de Paulin de Nole ${ }^{14}$, ainsi que dans le lectionnaire arménien ${ }^{15}$, qui a gardé en mémoire la liturgie de Jérusalem en usage vers 415 . On en trouve encore la trace dans un récit de pèlerin italien de 570 : l'Itinerarium Antonini Placentini ${ }^{16}$.

12 Ibid., XXXVII, 1-3: «On place alors un siège pour l'évêque au Golgotha, derrière la Croix, où il se tient à ce moment-là. L'évêque s'assied sur ce siège et l'on dispose devant lui une table couverte d'une nappe. Autour de la table, les diacres se tiennent debout. On apporte le coffret d'argent doré qui contient le saint bois de la croix, on l'ouvre, on l'expose, on place sur la table et le bois de la croix et l'écriteau. [...] Quand on les a placés sur la table, l'évêque, assis, appuie de ses mains sur les extrémités du bois sacré, et les diacres, debout tout autour, surveillent. Voici pourquoi cette surveillance. Il est d'usage que tout le peuple, tant fidèles que catéchumènes, s'approche un à un, se penche sur la table, baise le bois sacré et passe. Or on raconte que quelqu'un, je ne sais quand, y a mordu et a volé un fragment du bois sacré. C'est pourquoi maintenant les diacres, debout à l'entour, surveillent ainsi, pour qu'aucun de ceux qui approchent n'ose refaire de même. [...] Tout le peuple défile donc un à un. Chacun s'incline, touche du front, puis des yeux, la croix et l'écriteau, baise la croix et passe, mais personne n'étend la main pour toucher. Lorsqu'on a baisé la croix et qu'on est passé, un diacre est là debout qui tient l'anneau de Salomon et l'ampoule de l'onction des rois. On baise l'ampoule ; on vénère l'anneau [...] à la sixième heure, tout le peuple défile, entrant par une porte, sortant par une autre, car cela a lieu à l'endroit où, la veille, le jeudi, on a fait l'oblation ».

${ }^{13}$ Henri Leclercq, «Croix et crucifix », art. cit. ; Sebastia Janeras, Le Vendredi Saint dans la tradition liturgique byzantine: structure et histoire de ses offices, Rome, Pontificio ateneo S. Anselmo, coll. «Studia Anselmiana» $\mathrm{n}^{\circ} 99$ et «Analecta liturgica $», \mathrm{n}^{\mathrm{0}} 12,1988$, p. 280.

${ }^{14}$ Paulin de Nole. Ep. XXXI, 6, PG 61, p. 329-330. Sebastia Janeras, Le Vendredi Saint dans la tradition liturgique byzantine, op. cit., p. 282.

${ }^{15}$ Ibid., p. 283.

${ }^{16}$ Ibid., p. 283. 
Cette fête s'inscrit dans le cycle des fêtes christiques, autour de Noël et de Pâques. Liée à cette dernière, elle est également mobile : fête proprement chrétienne, elle semble fondée sur l'Évangile, sans emprunt extérieur, contrairement à de nombreuses célébrations chrétiennes.

La pratique de l'ostension de la Croix lors du Vendredi saint prend fin à Jérusalem en 614 avec le pillage de la ville par les Perses et le départ des reliques. Elle ne fut pas rétablie malgré le retour du bois de la Croix en $630^{17}$. En 635, Héraclius emmène les reliques de la Croix à Constantinople et réinstaure probablement à cette occasion la pratique de l'adoration de la Croix lors du Vendredi saint, même si ce n'est que vers 670 que l'on trouve le premier témoignage d'une cérémonie où la Croix était exposée pour être vénérée ${ }^{18}$. Trois jours de vénération à SainteSophie furent instaurés : le jeudi pour l'empereur et les hommes, le vendredi pour l'impératrice et les femmes, le samedi pour le patriarche et le clergé. On retrouve les mêmes informations sous la plume de Bède dans sa description de la Terre sainte : De locis sanctis, en 702 ou $703^{19}$. En revanche, l'adoration du Vendredi saint disparut au $\mathrm{X}^{\mathrm{e}}$ siècle, reportée à la troisième semaine de carême ${ }^{20}$ et au 14 septembre, cette dernière devenant une fête de plus en plus importante.

\footnotetext{
${ }^{17}$ Henri Leclercq, «Croix et crucifix », art. cit. ; Pierre Jounel, « Le culte de la Croix dans la liturgie romaine », La Maison Dieu, 75, 1963, p. 68-91, cit. p. 83.

${ }^{18}$ Sebastia Janeras, Le Vendredi Saint dans la tradition liturgique byzantine, op. cit., p. 290. Louis van Tongeren, Exaltation of the Cross, op. cit., p. 3.

${ }^{19}$ Sebastia Janeras, Le Vendredi Saint dans la tradition liturgique byzantine, op. cit., p. 290. Hector Quilliet, «Croix (adoration de la)», dans Dictionnaire de théologie catholique, éd. Alfred Vacant, Eugène Mangenot et Emile Amann, Paris, Letouzey et Ané, 1923, t. III, $2^{\mathrm{e}}$ partie, col. 2339-2363, cit. col. 2345.

20 «La commémoration et vénération de la Croix la troisième semaine du Carême semble être en étroite relation avec la reposition de la vraie Croix à Jérusalem par Héraclius. Nous avons déjà vu que cela avait eu lieu le Vendredi saint, 21 mars, de l'année 631. D'autres traditions attribuent ce fait aux premiers jours du mois de mars 630, précisément à la troisième semaine du Carême. C'est ainsi que cette vénération de la croix aurait pris son origine. Le lectionnaire géorgien, pour Jérusalem, ne connaît pas encore cette commémoraison. Dans la capitale byzantine elle apparut
} 
À Jérusalem cette fête repose sur la présence de reliques. Cette matérialité de l'adoration explique que longtemps la fête resta locale, tout comme elle explique son développement à Constantinople après l'installation de la relique. En revanche l'implantation de la fête en Occident montre que l'adoration ne porte plus sur la relique, mais sur le symbole qu'elle représente : n'importe quelle croix peut recevoir cette adoration. À Rome, l'adoration du Vendredi saint est la dernière des trois fêtes que nous étudions à s'implanter. Les premières attestations du rite se trouvent au début du $\mathrm{VIII}^{\mathrm{e}}$ siècle et le déroulement de la cérémonie témoigne de son origine orientale. Un reliquaire contenant le bois de la Croix est porté du Latran au palais Sessorien par un diacre, précédé par le pape, pieds nus, avec un encensoir. Cet ordre est typique de la pratique orientale, là où les pratiques romaines auraient inversé l'ordre de la procession $^{21}$. Il est curieux de constater que cette pratique arrive à Rome alors même qu'elle a peut-être déjà changé de date à Constantinople. Le modèle dont s'inspire le pape n'est d'ailleurs pas tant celui de Byzance que celui de Jérusalem et la liturgie de l'époque se caractérise par cette volonté de copier la liturgie de la Ville sainte ${ }^{22}$.

On le voit par exemple dans la place importante accordée à la Croix dans la procession vespérale de Pâques ${ }^{23}$. Il s'agit d'une reprise des pratiques de Jérusalem telles que les décrit Égérie : chaque soir de l'octave pascale, le clergé et le peuple se réunissaient avec les nouveaux baptisés autour de l'évêque sur le Mont des Oliviers. La procession effectuait une première station à l'église de l'Éléona, une deuxième dans l'église de l'Ascension, puis redescendait à l'Anastasis. Ainsi, à Rome,

probablement vers le VIII ${ }^{\mathrm{e}}$ siècle. Germain de Constantinople la connaît déjà, mais pas encore Arculphe. » Sebastia Janeras, Le Vendredi Saint dans la tradition liturgique byzantine, op. cit., cit. p. 291, voir également p. 298.

${ }^{21}$ Pierre Jounel, «Le culte de la Croix dans la liturgie romaine », art. cit., cit. p. 83.

22 Ibid.

${ }^{23}$ Ibid., cit. p. 86. 
le peuple se réunissait le soir de Pâques dans la basilique du Latran, autour des nouveaux baptisés, puis une procession le conduisait au baptistère et à l'oratoire Sainte-Croix. Le but de cette cérémonie était de marquer de façon solennelle l'entrée dans l'Église des néophytes. La cérémonie romaine rencontra un certain succès en France et en Allemagne où elle fut reprise même dans des lieux où la configuration de l'espace ne permettait pas le déplacement des fidèles.

\section{Exaltatio crucis : une date pour de multiples commémorations}

L'exaltation de la Croix constitue l'une des douze grandes fêtes du calendrier liturgique de l'Église d'Orient. À Jérusalem, elle s'étendait sur huit jours à compter du 13 septembre. Au $\mathrm{V}^{\mathrm{e}}$ siècle, on y ajoute une exposition de la Croix dans l'église du Martyrium, le 14 septembre.

$\mathrm{Si}$ aujourd'hui on l'associe au retour des reliques de la Croix en 628 sous Héraclius, cette fête apparaît plus tôt, dès la deuxième moitié $\mathrm{du} \mathrm{IV}^{\mathrm{e}}$ siècle, mais elle prend un surcroît de sens au VII ${ }^{\mathrm{e}}$ siècle. La première mention de cette fête se trouve dans le manuscrit de la lettre d'Égérie ${ }^{24}$ qui s'interrompt au moment où cette dernière décrit le déroulement des Encennies, fête de dédicace du Martyrium, église construite par Constantin à l'emplacement de la tombe du Christ. Égérie explique que les cérémonies qui célèbrent les dédicaces du Martyrium et de la chapelle proche, l'Anastasis, ont lieu en même temps car les deux églises auraient été consacrées le même jour ${ }^{25}$. Sur ce dernier point, Égérie est dans l'erreur. Le Martyrium a été consacré en 335, trentième

\footnotetext{
${ }^{24}$ Égérie, Journal de voyage, op. cit., cit. p. 316-317.

25 «On appelle jour de la dédicace celui où la sainte église qui est au Golgotha, celle qu'on appelle le Martyrium, a été consacrée à Dieu. La sainte église qui se trouve à l'Anastasis, à l'endroit où le Seigneur est ressuscité après sa passion, a été elle aussi consacrée à Dieu le même jour », traduction de Pierre Maraval, Égérie, Journal de voyage, ibid.
} 
année du règne de Constantin après le concile de Tyr, mais l'Anastasis n'aurait été achevée que plus tardivement ${ }^{26}$. D'après Égérie, la célébration, qui dure huit jours, est très solennelle car la Croix du Christ a été retrouvée le jour même des dédicaces, toutefois elle ne comporte pas d'ostension de la $\operatorname{Croix}^{27}$. On retrouve par la suite la fête codifiée dans la version la plus ancienne du lectionnaire arménien ${ }^{28}$.

La date de la dédicace fut choisie par Constantin qui ordonna aux évêques présents au concile de Tyr d'y assister ${ }^{29}$. Le choix de la date ne saurait donc être anodin et de nombreuses hypothèses peuvent l'expliquer, sans s'exclure forcément l'une l'autre. D'après Michael Fraser et David Hunt ${ }^{30}$, les ides de septembre (soit le 13 du mois) seraient la date de l'epulum Jovis in Capitolio, qui pouvait passer pour le jour de la fête de la dédicace du temple de Jupiter capitolin à Rome. Sous l'empereur Hadrien, la ville de Jérusalem fut détruite suite à la révolte de Bar Kokhba (132-135) et refondée sous le nom d'Aelia capitolina. Un sanctuaire en l'honneur de Jupiter fut alors construit à l'emplacement du tombeau du Christ. Des réjouissances en l'honneur de la dédicace de ce temple devaient également se tenir le 13 septembre sur le modèle romain.

\footnotetext{
${ }^{26}$ Henri Leclercq, « Croix et crucifix », art. cit., cit. col. 2347 ; Louis van Tongeren, Exaltation of the Cross, op. cit., p. 1 ; Pierre Maraval, Lieux saints et pèlerinages d'Orient : histoire et géographie des origines à la conquête arabe. Dictionnaire des lieux saints, Paris, Éditions du Cerf, coll. « Histoire », $2004^{2}$ (1985), cit. p. 252-256.

27 Henri Leclercq, «Croix (invention et exaltation de la vraie)», art. cit., cit. col. 3131 ; Pierre Jounel, «Le culte de la Croix dans la liturgie romaine », art. cit., cit. p. 71 ; Louis van Tongeren, Exaltation of the Cross, op. cit., cit. p. 18.

${ }^{28}$ Lectionnaire arménien cité par Pierre Jounel, « Le culte de la Croix dans la liturgie romaine », art. cit., p. 71 et Louis van Tongeren, Exaltation of the Cross, op. cit., cit. p. 17.

${ }^{29}$ Michael F. Fraser, «Constantine and the Encaenia », Studia patristica, 29, 1997, p. 25-28, cit. p. 25.

30 E. D. Hunt, «Constantine and Jerusalem », Journal of Ecclesiastical History, vol. $48, \mathrm{n}^{\mathrm{o}} 3$, juillet 1997 , p. 405-432, cit. p. 421.
} 
Il se pourrait aussi que le 13 septembre soit la date anniversaire de la victoire de Constantin sur un autre de ses concurrents, Lucinius, en $324^{31}$. Égérie signale que la fête des Encennies célébrait également la dédicace du Temple de Jérusalem par Salomon ${ }^{32}$. Le rapprochement entre Constantin bâtissant une nouvelle église à Jérusalem et le roi Salomon se trouve à plusieurs reprises, par exemple sous la plume d'Eusèbe qui qualifie le Martyrium de «nouvelle Jérusalem ${ }^{33}$ ». Chez les Juifs, la fête de la dédicace du Temple, ou Yom Kippour, est célébrée le $10 \mathrm{du}$ mois de Tishri qui tombait en 335 le 13 septembre $^{34}$. Notons d'ailleurs qu'avant d'être utilisé pour la dédicace du Martyrium, le terme des Encennies désigne la fête de Hanoucca qui commémore la nouvelle inauguration de l'autel des offrandes dans le second Temple de Jérusalem, lors de son retour au culte judaïque, trois ans après son interdiction par le Séleucide Antiochus IV. Le choix de la date s'inscrirait ainsi à la fois dans le contexte politique de l'empire en 335 et dans les traditions de célébration de la ville de Jérusalem, tant romaine que juive.

En Orient, le culte de la Croix fut relancé par les événements du début du VII ${ }^{\mathrm{e}}$ siècle. Au cours de la guerre qui opposa les Perses aux Byzantins de 602 à 628, Jérusalem fut assiégée et prise en $614^{35}$. Les Perses détruisirent l'Anastasis et emportèrent les reliques de la Croix dans leur capitale, Ctésiphon. En 627, après la victoire de Ninive, Héraclius contraignit le chef des Perses, Kavadh II, à lui restituer la relique de la Croix. Peu après, il obtint également, de Schahr-Barâz, le retour de la sainte Éponge. Ces deux reliques furent apportées à

\footnotetext{
${ }^{31}$ Michael F. Fraser, « Constantine and the Encaenia », art. cit., cit. p. 26.

${ }^{32}$ Égérie, Journal de voyage, op. cit. ; E. D. Hunt, «Constantine and Jerusalem », art. cit., cit. p. 422.

${ }^{33}$ Michael F. Fraser, «Constantine and the Encaenia », art. cit., cit. p. 27.

${ }^{34}$ Ibid.

${ }^{35}$ Hector Quilliet, « Croix (adoration de la)», art. cit., cit. col. 2346.
} 
Constantinople, où elles firent l'objet d'une cérémonie que la tradition date du 14 septembre 629. La sainte Lance fut apportée dans la capitale byzantine le 28 octobre par Nicétas, fils de Schahr-Barâz, qui devait devenir patrice et résider à Constantinople. Elle fit à son tour l'objet de quatre jours de vénération publique du $1^{\mathrm{er}}$ au 4 novembre $629^{36}$.

Héraclius se rendit à Jérusalem au début de l'année 630 pour y rapporter la relique de la vraie Croix. Il y fut accueilli le 21 mars par Modeste de Jérusalem qui détenait le patriarcat depuis l'exil du patriarche Zacharie. Il entra dans la ville à pied, sans aucun insigne impérial, en portant lui-même la relique tout au long de la Via Dolorosa jusqu'au Calvaire. À cette occasion, il restaura l'église du SaintSépulcre. Grâce à Héraclius, la Croix fut à nouveau associée à l'empereur byzantin qui en distribuait des fragments en guise de faveur.

Bien que la fête de l'Exaltation de la Croix soit d'origine hiérosolymitaine, elle fut dès son origine associée à l'empereur. Une seconde fête célébrant la Croix à Jérusalem avait lieu le 7 mai pour commémorer l'apparition de la Croix à l'époque de Cyrille ${ }^{37}$ et relèverait davantage de l'initiative de l'évêque local.

À Rome, la date du 14 septembre était traditionnellement consacrée aux saints martyrs Corneille et Cyprien. La fête de l'Exaltation de la Croix arriva entre 650 et 680 à Rome où elle n'était d'abord célébrée que par la colonie grecque et les communautés orientales ${ }^{38}$. La

\footnotetext{
${ }^{36}$ Edina Bozóky, La Politique des reliques de Constantin à Saint Louis : protection collective et légitimation du pouvoir, Paris, Beauchesne, coll. «Bibliothèque historique et littéraire », 2007, cit. p. 21.

${ }^{37}$ Henri Leclercq, « Croix et crucifix », art. cit., col. 3136. Bernard Cornet, « La fête de la Croix du 3 mai », Revue belge de philologie et d'histoire, vol. 30, $\mathrm{n}^{\circ}$ 3-4, 1952, p. 837-848, cit. p. 839. Louis van Tongeren, Exaltation of the Cross, op. cit., cit. p. 3.

38 Antoine Chavasse, Le Sacramentaire Gélasien (Vaticanus Reginensis 316): sacramentaire presbytéral en usage dans les titres romains au VIIe siècle, Tournai,
} 
cérémonie se déroulait de la façon suivante: le prêtre élevait un fragment du bois de la Croix au-dessus de sa tête, puis il bénissait l'assemblée en se tournant vers les quatre points cardinaux tandis que le peuple chantait un kyrie eleison ${ }^{39}$. La fête passe dans les pratiques occidentales à la fin du VII siècle. À cette époque, le sacramentaire grégorien de Padoue ajoute à la fête des saints Cyprien et Corneille une oraison ad crucem salutandam in sancto Petro ${ }^{40}$. Au même moment, le pape Serge (687-701) redécouvre une relique de la Croix qui aurait été oubliée dans la sacristie de la basilique du Vatican ${ }^{41}$. L'intensification du culte pour la sainte Croix au début du VII ${ }^{\mathrm{e}}$ siècle peut s'expliquer par la situation de tension qui existe à Jérusalem et par les mouvements des reliques de la Croix. La fête est mentionnée dans le sacramentaire gélasien $^{42}$ et dans des évangéliaires romains du VII ${ }^{\mathrm{e}}$ siècle ${ }^{43}$. Le caractère récent de cette fête se perçoit dans un des manuscrits qui propose la même lecture pour l'Exaltation de la Croix que pour l'Inventio ${ }^{44}$. À partir du VIII siècle, la fête apparaît dans la plupart des livres liturgiques romains. Toutefois jusqu'au XIV ${ }^{\mathrm{e}}$ siècle, on continua de célébrer le même jour les martyrs Cyprien et Corneille et l'Exaltation de la Croix au cours

Desclée, coll. «Bibliothèque de Théologie, $4^{\mathrm{e}}$ série : Histoire de la théologie », $\mathrm{n}^{\mathrm{o}} 1$, 1958 , t. 1, cit. p. 361.

${ }^{39}$ Pierre Jounel, « Le culte de la Croix dans la liturgie romaine », art. cit., cit. p. 75.

${ }^{40}$ Sacramentaire de Padoue : Leo Cunibert Mohlberg, Leo Eizenhöfer, Petrus Siffrin, Liber sacramentorum Romanae Aeclesiae ordinis anni circuli (Cod. Vat. Reg. Lat. 216), Roma, Herder, coll. «Rerum ecclesiasticarum documenta. Series maior, Fontes $», n^{\circ} 4,1960, n^{\circ} 421-423$.

${ }^{41}$ Liber Pontificalis, éd. Theodor Mommsen, Berlin, Weidman, coll. «Monumenta Germaniae Historica, Gesta Pontificum Romanum », $\mathrm{n}^{\mathrm{0}} 1$, 1898, t. 1, cit. p. 374. Pierre Jounel, «Le culte de la Croix dans la liturgie romaine », art. cit., cit. p. 75.

${ }^{42}$ Pierre Jounel, «Le culte de la Croix dans la liturgie romaine », art. cit., cit. p. 76. Édition du sacramentaire gélasien: Leo Cunibert Mohlberg, Das fränkische Sacramentarium Gelasianum in alamannischer Überlieferung (Codex Sangall. $N$ 348) : St Galler Sakramentar-Forschungen I. Nachtrag. Münster, Aschendorff, coll. «Liturgiewissenschaftliche Quellen und Forschungen », $\mathrm{n}^{\circ} 1-2,1971^{3}$ (1918).

${ }^{43}$ Pierre Jounel, «Le culte de la Croix dans la liturgie romaine », art. cit., cit. p. 76.

${ }^{44}$ Pierre Jounel, «Le culte de la Croix dans la liturgie romaine », art. cit., cit. p. 75. Louis van Tongeren, Exaltation of the Cross, op. cit., p. 4. 
de deux messes successives. Ce n'est que sous le pape Grégoire XI (1370-1378) que la fête des martyrs fut transférée au 16 septembre ${ }^{45}$, ce qui montre bien le caractère secondaire que conserva longtemps la fête de l'Exaltation de la Croix en Occident.

\section{L' Inventio crucis, une création occidentale}

Tout au long du Moyen Âge, l'Inventio crucis occupa une place très importante dans les célébrations du mois de mai, avant d'être supplantée au XVI ${ }^{\mathrm{e}}$ siècle par le culte de la Vierge. La fête du 3 mai, qui célèbre la découverte du bois de la Croix, s'inscrit dans le cycle de Pâques où elle est la seule célébration à date fixe. C'est une particularité de l'Occident puisque l'invention de la Croix est célébrée en Orient en même temps que l'Exaltation. En revanche nous avons déjà noté l'existence d'une autre fête concernant la Croix au mois de mai, le 7.

On a longtemps considéré à la suite de Louis Duchesne ${ }^{46}$ et de Michel Andrieu ${ }^{47}$ que la fête de l'inventio crucis était d'origine gauloise car les attestations les plus anciennes figurent dans la liturgie gallicane et plus particulièrement dans le sacramentaire gélasien. Cependant Antoine Chavasse a montré grâce à une étude précise de la filiation entre les différentes familles de sacramentaires que cette fête était d'origine romaine $^{48}$.

On trouve la première attestation de la fête de l'Inventio crucis dans le Liber pontificalis, l'histoire des papes, dans la notice du pape

\footnotetext{
${ }^{45}$ Pierre Jounel, « Le culte de la Croix dans la liturgie romaine », art. cit., cit. p. 79.

${ }^{46}$ Louis Duchesne, Le Liber pontificalis. Texte, introduction et commentaire, Paris, Éditions de Boccard, coll. «Bibliothèque des Écoles françaises d'Athènes et de Rome », $\mathrm{n}^{\mathrm{0}} 1-3,3$ vol., $1981^{3}\left(1886-1892^{1}\right)$.

47 Les Ordines romani du haut Moyen Âge. Les manuscrits, éd. Michel Andrieu, Louvain, «Spicilegium sacrum Lovaniense. Études et documents », $\mathrm{n}^{\mathrm{o}} 11,1965^{2}$ $\left(1931^{1}\right)$.

${ }^{48}$ Antoine Chavasse, Le Sacramentaire Gélasien, op. cit., cit. p. 360.
} 
Serge (687-701) ${ }^{49}$. Malgré le terme d'exaltatio ici employée, il faut comprendre qu'il s'agit de la fête de l'inventio, qui apparait en première dans les livres de messe.

Le choix de la date du 3 mai pour célébrer l'invention de la Croix en Occident a connu plusieurs justifications. Dans le bréviaire romain, la fête est présentée comme l'anniversaire de la découverte de la Croix, alors qu'en Orient cette fête a lieu, comme nous l'avons vu, le 14 septembre en même temps que l'Exaltation de la Croix. D'autres ont rappelé que le 3 mai correspondait à un des épisodes du recouvrement de la Croix par l'empereur Héraclius. Plusieurs hypothèses ont été avancées : il pourrait s'agir, selon les auteurs, du jour où les Perses ont rendu la Croix à l'empereur, du jour où la relique arriva à Constantinople, ou encore du jour où Héraclius la restitua à Jérusalem ${ }^{50}$. Ces hypothèses sont peu étayées car ces événements devaient sembler fort lointains pour les Occidentaux et nous n'avons aucune trace d'une fête de la Croix en Orient, le 3 mai.

Bernard Cornet propose une élaboration plus complexe et tout à fait séduisante. La célébration de la fête au 3 mai reposerait sur une confusion de la part des Occidentaux qui auraient eu connaissance de la fête orientale célébrée le 7 mai, parvenue en Occident avec la légende de Judas Cyriaque. Il s'agit d'un récit de la découverte de la Croix où un Juif, Judas, joue le rôle principal au côté de l'impératrice Hélène. Ce texte connut un grand succès et fut diffusé en grec, en syriaque et en latin. Bernard Cornet considère que le nom de la fête, apparitio, n'aurait

\footnotetext{
${ }^{49}$ Liber Pontificalis, op. cit, cit. p. 213 : «Qui etiam ex die illo pro salute humani generis ab omni populo christiano, die Exaltationis sanctae Crucis, in basilicam Salvatoris, quae appellatur Constantiniana osculatur ac adoratur »; «Et en ce jour, pour le salut du genre humain, jour de l'Exaltation de la sainte Croix, dans la basilique du Sauveur que l'on appelle constantinienne, elle est vue et adorée par tout le peuple chrétien » (nous traduisons).

${ }^{50}$ Bernard Cornet, «La fête de la Croix du 3 mai », art. cit., cit. p. 838.
} 
jamais été compris par les Occidentaux qui l'auraient associé la date à l'inventio de la Croix dont on trouve le récit dans la légende de Judas Cyriaque $^{51}$. Dans un second temps, un glissement se serait produit entre le 7 et le 3 mai. Bernard Cornet l'explique en supposant une difficulté dans la lecture de la date. Le VII mai aurait été mal interprété par un copiste qui aurait lu VN mai, sachant que les nones pouvant être abrégée par un $\mathrm{N}$ majuscule ${ }^{52}$.

Cette ingénieuse hypothèse n'est pas sans poser de problèmes. D'une part, l'association entre la légende de Judas Cyriaque à la fête de l'Apparitio n'est attestée ni en Orient, ni en Occident. D'autre part, si on trouve, de façon occasionnelle, la date du 7 mai en Occident, en particulier dans le codex epternacensis ${ }^{53}$, cela ne nous prouve pas que l'erreur ne venait pas de ce copiste. On trouve en effet dès le vi siècle, donc bien avant ce codex, dans le Liber Pontificalis, la date du 3 mai pour la découverte de la Croix. À l'évidence, le Liber Pontificalis s'inspire de la légende de Judas Cyriaque dont la version latine s'achève ainsi : «Helena dormivit in pace [...] demandans omnibus qui Christum diligunt, viris ac milieribus, celebrare comemorationem diei in qua inventa est sancta crux, quinto ninarum Maiarum ${ }^{54}{ }$. Cette précision est propre à la tradition latine du texte et ne se trouve pas dans les versions

\footnotetext{
${ }^{51}$ Ibid., cit. p. 842.

${ }^{52}$ Ibid., cit. p. 846.

${ }^{53}$ Le codex epternacensis. BnF ms. lat. 10837 est le plus ancien représentant du martyrologe hiéronymien et le plus proche de l'archétype, on le date des alentours de 750.

54 «Hélène s'endormit en paix [...], demandant à tous, hommes et femmes, qui sont zélés pour le Christ, de célébrer la commémoration du jour où la sainte Croix a été inventée, le 3 mai ». Liber Pontificalis, cit. p. 167.
} 
syriaque ou grecque ${ }^{55}$. Elle semble n'avoir été ajoutée que parce que la légende devait servir de lecture liturgique.

Une autre hypothèse nous vient des Bollandistes selon lesquels le 3 mai commémorait l'anniversaire du jour où un important fragment de la Croix serait arrivé à Rome à l'initiative de l'impératrice Hélène ${ }^{56}$. Cette dernière aurait fait déposer ces reliques dans la chapelle qui jouxtait sa résidence, le palais Sessorien, et qui avait pour vocation de commémorer la victoire de son fils au Pont Milvius. La fête aurait d'abord été célébrée exclusivement dans cette basilique avant de se répandre lentement dans Rome. Malheureusement aucune tradition de la fin de l'Antiquité ou du haut Moyen Âge n'étaie cette hypothèse.

Des rapprochements avec le calendrier romain peuvent expliquer en partie le choix de la date du 3 mai. En effet, du 28 avril au 3 mai avaient lieu les Floralies, fêtes dédiées à Flore, bien connues grâce aux Fastes d'Ovide ${ }^{57}$ et célèbres pour leur licence. On peut donc envisager que comme pour la Saint-Valentin, qui est une christianisation des fêtes non moins scandaleuses des Lupercales par le pape Gélase $\mathrm{I}^{\mathrm{er}}(495)^{58}$, on ait tenté ici de christianiser les fêtes des Floralies.

Ces célébrations n'étaient pas les seules fêtes d'envergure au début du mois de mai. Les Celtes célébraient Beltaine, fête sacerdotale fondée sur le cérémonial du sacre et qui marque la fin de la saison sombre et le début de la saison claire ${ }^{59}$. Ces deux fêtes, les Floralies et Beltaine, qui célébraient le retour des beaux jours et de la pleine activité

\footnotetext{
55 Bernard Cornet, «La fête de la Croix du 3 mai », art. cit., cit. p. 846 ; Antoine Chavasse, Le Sacramentaire Gélasien, op. cit., cit. p. 353 ; Pierre Jounel, « Le culte de la Croix dans la liturgie romaine », art. cit., cit. p. 172.

${ }^{56}$ Acta Sanctorum Maii (AASS Maii), Antverpiae, apud Michelem Cnobarum, 1680, t. I, p. 361.

${ }^{57}$ Ovide, Fastes, V.

${ }^{58}$ Philippe Walter, Mythologie chrétienne, op. cit.

59 Antoinette Glauser-Matecki, Un Aspect du cycle de mai en Europe occidentale, op. cit., cit. p. 8.
} 
agricole, ont certainement influencé les célébrations de l'Invention de la Croix. Ainsi, les coutumes et les fêtes situées dans les premiers jours de mai ont conservé les vertus magiques attribuées au coudrier par les Celtes d'Irlande ${ }^{60}$ : il convenait le 3 mai de faire bénir des baguettes de coudrier appelées les «Croisettes» que l'on plaçait ensuite dans les champs pour garantir une récolte fructueuse. On notera, au passage, qu'il convenait d'ôter ces croisettes le 14 septembre. Le lien semble également facile à établir entre la pratique des arbres de mai que l'on plantait devant les maisons de ceux que l'on voulait honorer, les légendes de «saints au bâton reverdissant » et la fête de l'invention de la sainte Croix $^{61}$. Le lien est explicité par exemple par la figure de saint Christophe qui personnifie, comme Porte-Christ ou Porte-Croix, le « reverdissement » dans les légendes chrétiennes.

On voit donc à Rome l'apparition de l'invention de la Croix, puis d'une fête de l'exaltation et enfin d'une adoration de la Croix le Vendredi saint. Par la suite, l'époque carolingienne, qui voit l'essor des dévotions à la $\mathrm{Croix}^{62}$, répandit ces fêtes à travers tout l'empire quand Charlemagne choisit de diffuser la liturgie romaine au détriment des pratiques gallicanes.

Deux fêtes distinctes supposent deux besoins différents qui s'ajoutent à la simple commémoration du Vendredi saint. Ma première hypothèse était que l'une des fêtes fut instituée par le pouvoir impérial et l'autre par le pouvoir pontifical. Si séduisante que soit cette idée, rien ne semble la prouver.

\footnotetext{
${ }^{60}$ Ibid., cit. p. $50-52$ et 361.

${ }^{61}$ Ibid., cit. p. 372 et 374.

${ }^{62}$ Celia Martin Chazelle, The Crucified God in the Carolingian era : theology and art of Christ's Passion, Cambridge/New York, Cambridge University Press, 2001 ; Marie-Christine Sepière, L'Image d'un Dieu souffrant $\left(I X^{e}-X^{e}\right.$ siècles) : aux origines du crucifix, Paris, Éditions du Cerf, coll. « Histoire », 1994.
} 
On constate aussi que les deux dates du 3 mai et du 14 septembre permettent un encadrement de l'année suivant le calendrier agricole. Traditionnellement, la chasse au vol s'étendait par exemple du 3 mai au 14 septembre tandis que la chasse aux bêtes commençait le 14 septembre et durait jusqu'au 3 mai $^{63}$. De même ces deux dates servaient de point de départ et de retour à la transhumance en Provence. On sait combien le calendrier agricole a influencé le calendrier liturgique, les périodes les plus intenses spirituellement étant aussi celles de moindre activité agricole. On sait aussi que l'Église nia toujours ce lien. On ne saurait non plus négliger les héritages antiques et païens dans la fixation du calendrier chrétien. Le haut Moyen Âge apparaît ainsi comme la période de la christianisation du temps de la fête.

${ }^{63}$ Antoinette Glauser-Matecki, Un Aspect du cycle de mai en Europe occidentale, op. cit., cit. p. 58. 